\title{
XXXVIII. On achromatic polarization and differential double refraction
}

\section{B. Brace Ph.D.}

To cite this article: D. B. Brace Ph.D. (1899) XXXVIII. On achromatic polarization and differential double refraction, Philosophical Magazine Series 5, 48:293, 345-360, DOI: 10.1080/14786449908621344

To link to this article: http://dx.doi.org/10.1080/14786449908621344

曲 Published online: 08 May 2009.

Submit your article to this journal $[\pi$

Џ Article views: 2

Q View related articles $\asymp$ 


\section{[ 345$]$}

XXXVIII. On Achromatic Polarization and Differential Double Refraction. By D. B. Brace, Ph.D., Professor of Physics, University of Nebraska*.

$\mathbf{I}^{\mathrm{T}}$

F a ray of light polarized at $45^{\circ}$ to the principal axes of a crystalline plate pass normally through it, the relative retardation of the components will be proportional to the thickness and to the difference in the refractive indices. In most crystals the differential double refraction in the visible spectrum is normal, and the relative retardation increases with the frequency, depending on the crystal. By crossing several such plates the difference in the resultant retardation for adjacent parts of the spectrum might be made a minimum.

The object of this investigation was to determine whether such minima existed, and what orders would give the best results. Of the crystals examined, namely, Iceland spar, quartz, selenite, mica, and aragonite, all wore found to give more or less perfect achromatism through the greater part of the visible spectrum.

If $d_{1}, \epsilon_{1}, \omega_{1}, d_{2}, \epsilon_{2}, \omega_{2}, \ldots$ are the lengths of the path and the reeiprocals of the velocities of the component rays in the successive plates, and $\lambda$ the wave-length in vacuo, the relative retardation or order $N$ is given by the equation

$$
d_{1} \frac{\epsilon_{1}-\omega_{1}}{\lambda}+d_{2} \frac{\epsilon_{2}-\omega_{2}}{\lambda}+d_{3} \frac{\epsilon_{3}-\omega_{3}}{\lambda} \ldots=\mathrm{N}, .
$$

or

where

$$
\mathrm{N}_{1}+\mathrm{N}_{2}+\mathrm{N}_{3} \ldots=\mathrm{N} ; \text {. . . . }
$$

$$
\mathrm{N}_{1}=d_{1} \frac{\epsilon_{1}-\omega_{1}}{\lambda}, \quad \mathrm{N}_{2}=d_{2} \frac{\epsilon_{2}-\omega_{2}}{\lambda} \ldots
$$

Hence

$$
\frac{\delta}{\delta \lambda}\left(d_{1} \frac{\epsilon_{1}-\omega_{1}}{\lambda}+d_{2} \frac{\epsilon_{2}-\omega_{2}}{\lambda}+\ldots\right)=\frac{\delta N}{\delta \lambda}=0 ; . .
$$

or

$$
\frac{\delta}{\delta \lambda}\left(\mathrm{N}_{1}+\mathrm{N}_{2}+\ldots .\right)=\frac{\delta \mathrm{N}}{\delta \lambda}=0 ; . . . .
$$

i.e. the difference in the relative retardation for two frequencies will be a very small quantity if the equation holds. If it can be satisfied thronghout the visible spectrum, perfect achromatism will be possible.

* Communicated by the Author. 
If $d_{1}, d_{2}, \ldots$ are approximately constant for the entire aperture

$$
d_{1} \frac{\delta\left(\frac{\epsilon_{1}-\omega_{1}}{\lambda}\right)}{\delta \lambda}+d_{2} \frac{\delta\left(\frac{\epsilon_{2}-\omega_{2}}{\lambda}\right)}{\delta \lambda}+\ldots=\frac{\delta \mathrm{N}}{\delta \lambda}=0 ;
$$

and hence

$$
d_{1} \frac{\delta\left(\epsilon_{1}-\omega_{1}\right)}{\delta \lambda}+d_{2} \frac{\delta\left(\epsilon_{2}-\omega_{2}\right)}{\delta \lambda}+\ldots=\mathrm{N} . . .
$$

Equation (3) gives the ratio of the paths in the several plates for achromatism in any part of the spectrum where equation (2) holds.

Equation (4) gives the length of the path in each crystal necessary for a resultant retardation of $\mathrm{N}$ wave-lengths for those rays which can be achromatized. The case of achromatic $1 / 4 \lambda, 1 / 2 \lambda, \ldots$ wave-plates will illustrate such compound achromatic systems.

If $d_{1}$ and $d_{2}$ are not constant over the entire aperture, then for any other thickness we must have as a consequence of $(\ddot{b})$

$$
\left(d_{1}+\delta d_{1}\right) \frac{\delta\left(\frac{\epsilon_{1}-\omega_{1}}{\lambda}\right)}{\delta \lambda}+\left(d_{2}+\delta d_{2}\right) \frac{\delta\left(\frac{\epsilon_{2}-\omega_{2}}{\lambda}\right)}{\delta \lambda}+\ldots=0
$$

Hence from (3)

$$
\delta d_{1} \frac{\delta\left(\frac{\epsilon_{1}-\omega_{1}}{\lambda}\right)}{\delta \lambda}+\delta d_{2} \frac{\delta\left(\frac{\epsilon_{2}-\omega_{z}}{\lambda}\right)}{\delta \lambda}+\ldots=0 .
$$

and thus

$$
\delta d_{1} \frac{\delta\left(\epsilon_{1}-\omega_{1}\right)}{\delta \lambda}+\delta d_{2} \frac{\delta\left(\epsilon_{2}-\omega_{2}\right)}{\delta \lambda}+\ldots=\delta \mathrm{N}
$$

It follows also that

$$
\frac{\delta d_{1}}{\delta l} \frac{\delta\left(\epsilon_{1}-\omega_{1}\right)}{\delta \lambda}+\frac{\delta l_{2}}{\delta l} \frac{\delta\left(\epsilon_{2}-\omega_{2}\right)}{\delta \lambda}+\ldots=\frac{\delta N}{\delta l}, \quad .
$$

where $\delta l$ is the distance between adjacent points in the field of view of a normal spectrum.

Equation (3b) shows that if at any point in the aperture there is achromatism, the same will be true at any other point if the variation in the paths have the same ratio as in (3).

Equation $(4 a)$ gives the variation in paths $\delta d_{1}, \delta d_{2}$ which will produce a variation of $\delta \mathrm{N}$ in the resultant order. If, for example, the system consists of a series of wedges and 
plates, a change of order $\delta \mathrm{N}$ at any point in the aperture may be produced by the variations found from this equation. If $\mathrm{N}$ is the sameover the entire aperture, then for any increments $\delta d_{1}, \delta d_{2}, \ldots$. the new order will be the same over the entire aperture. Such a system would be obtained by a combination of wedges or wedges and plates, giving white light of uniform intensity over the entire field, as in instruments constructed after the manner of the colour-compensators of Bravais, Biot, Soleil, and others.

Equation (5) gives the number of bands between two points in the aperture whose distance is $\delta l$ when the total path varies by $\delta d_{1}+\delta d_{2}+\ldots$ uniformly, and the surfaces of the plates are plane and inclined, as in the case of a compound system of wedges, or of wedges and plane parallel plates. The number of achromatic bands $\frac{\delta \mathrm{N}}{\delta l}$ visible in a unit length of the field in any direction would letermine the total variation in the paths $\frac{\delta d_{1}+\delta d_{2}+\ldots}{\delta l}$. per unit distance for the entire system. If the total variation in path in any direction for a unit length is given, the number of achromatic bands visible in a unit distance of the aperture in this direction can be determined. An example of such a conipound system giving achromatic bands would be an optical compensator similar in construction and effect to one of the forms of Babinet's compensator used with monochromatic light. Both in the compensators with interferencebands and in those with uniform field, the calibration giving the value of $\delta \mathrm{N}$ (the variation in order) due either to a displacement of the plates or to a change of the point of reference in the field equal to $\delta l$ is obtained from equation (5).

From equation (2a) we have for two crystals

$$
\frac{\delta N_{1}}{\delta \lambda}+\frac{\delta N_{2}}{\delta \lambda}=0 \text {, }
$$

or

$$
\frac{\delta \mathrm{N}_{1}}{\delta \lambda}=-\frac{\delta \mathrm{N}_{2}}{\delta \lambda}, \quad . \quad \cdot \quad \cdot \quad \cdot .
$$

as the condition of achromatism. This indicates that if in the two crystals the orders either increase or decrease with the frequency they are to be crossed or placed in subtractive series. If, however, in one the orders increase and in the other they decrease with the frequency, they are to be placed parallel or in additive series.

This immediately suggests an experimental method of 
testing various combinations for achromatism. Müller * first studied interference in crystalline plates by means of spectrum analysis. Fizeau and Foucault $\dagger$ and others have also studied the same phenomena in crystals taken singly. The observations of Mouton $\ddagger$ on the differential double refraction in a plate of selenite indicate anomalous dispersion at $491 \mu$, that is, $\epsilon-\omega$ is a minimum in this region. Later observations by Dufet $\$$ indicate, on the contrary, that it is normal, and follows the law of Briot in the visible spectrum, as do quartz and Iceland spar. The observations of Mouton should agree with the latter's observations if the retardation in the plate used by Mouton were $9 \lambda$ instead of $10 \lambda$ according to his measurements.

In all such combinations then the plates must be placed in subtractive order. How far the conditions of (6) are fuJfilled by crystals already examined is not readily determined from present available data.

Since $\frac{\delta \mathrm{N}_{1}}{\delta \lambda}, \frac{\delta \mathrm{N}_{2}}{\delta \lambda}$ are the number of bands in the spectrum between the wave-lengths $\lambda$ and $\lambda+\delta \lambda$, it must be possible by varying the length of the path to make this number the same throughout the spectrum, in order to obtain perfect achromatism.

For this purpose direct comparisons of the interferencebands in two crystals were made. The displacement of the bands and the increase in their number, and also the determination of the order at any point in the spectrum, may be anticipated from the law for differential double refraction. Assuming as the law for differential double refraction, Briot's law for dispersion-

$$
\epsilon-\omega=\mathrm{A}+\frac{\mathrm{B}}{\lambda^{2}}+\frac{\mathrm{C}}{\lambda^{4}}-\delta \lambda^{2},
$$

where $A, B, C, D$ are all positive quantities, we have

$$
\mathrm{N}=d \frac{\epsilon-\omega}{\lambda}=\frac{d}{\lambda}\left(\mathrm{A}+\frac{\mathrm{B}}{\lambda^{2}}+\frac{(j}{\lambda^{2}}-\delta \lambda^{2}\right) . \quad .
$$

Hence

$$
\frac{\delta \mathrm{N}}{-\delta \lambda}=\frac{d}{\lambda^{2}}\left(\mathrm{~A}+\frac{3 \mathrm{~B}}{\lambda^{2}}+\frac{5 \mathrm{C}}{\lambda^{4}}-\delta \lambda^{2}\right), \quad .
$$

also

$$
\frac{\delta}{-\delta \lambda} \frac{\delta \mathrm{N}}{(-\delta \lambda)}=\frac{d}{\lambda^{3}}\left(2 \mathrm{~A}+\frac{12}{\lambda^{2}}+\frac{30 \mathrm{C}}{\lambda^{4}}\right) . .
$$

* Pogg. Ann. vol. lxix. p. 98.

+ Ann. de Chim. et Phys. (3) t. xxvi. p. 145 ; t. xxx. p. 148.

+ Comptes Rendus, t. Ixxxviii. pp. 1087, 1189 (1879).

Journal de Physique, 1888, pp. 305-306. 
Equation (8) shows that the order increases toward the violet end of the spectrum, and equation (9) that the number of bands per unit distance in the normal spectrum increases as the wave-length diminishes. We have also from equation (7), if $N$ is constant and $d$ and $\lambda$ vary,

$$
\frac{\delta \lambda}{\delta d}=\frac{\mathrm{N}}{d} \cdot \frac{1}{\frac{d}{\lambda^{2}}\left(A+\frac{3 \mathrm{~B}}{\lambda^{2}}+\ldots\right)}, \quad \cdot . .
$$

and also

$$
\frac{\delta \mathrm{N}}{\delta \bar{d}}=\frac{1}{\lambda}\left(\mathrm{A}+\frac{\mathrm{B}}{\lambda^{2}}+\frac{\mathrm{C}}{\lambda^{l}}-\delta \lambda^{2}\right) . \quad . \quad .
$$

In equation (10) the second member is a positive quantity, and hence if $d$ increases $\lambda$ must increase, and consequently the position of a band of any order must be shifted toward the red end of the spectrum when the length of the path in the crystal is increased. The direction of displacement prodnced by increase of $d$ is taken as the positive direction. In equation (11), if we take $\lambda$ constant (say for one of the sodium lines) we have the number of bands passing this point from the violet toward the red end of the spectrum for any increase in the path. Furthermore, since the righthand side of the equation increases as $\lambda$ diminishes, we shall have more bands coming into the vinlet end of the spectrum than disappear at the red end, and hence the number of bands visible will increase with $d$.

It follows at once from the above interpretation that we can determine the order of the plate for any wave-length by counting the number of bands passing this point when $d$ is diminished or increased, until new coincidences in a different ratio obtain throughout the spectrum.

Thus we have

and

$$
\frac{\delta \mathrm{N}}{-\delta \lambda}=\frac{d}{\lambda^{2}}\left(\mathrm{~A}+\frac{3 \mathrm{~B}}{\lambda^{2}} \ldots\right)
$$

$$
\frac{\delta \mathrm{N}^{\prime}}{-\delta \lambda}=\frac{d^{\prime}}{\lambda^{\overline{2}}}\left(\mathrm{~A}+\frac{3 \mathrm{~B}}{\lambda^{4}} \ldots\right) .
$$

The first equation gives the intervals $-\delta \lambda$ in wave-length for the same increment $\delta \mathrm{N}$ in the order, for path $d$ throughout the spectrum, and the second equation similarly the increment $\delta N^{\prime}$ in the order for a path $d^{\prime}$, so that we may have a coincidence of bands throughout the second spectrum at the same intervals $\delta \lambda$. 
From these equations we obtain

$$
\frac{\frac{\delta N}{\delta \bar{\lambda}}}{\frac{\delta N^{\prime}}{\delta \lambda}}=\frac{d}{d^{\prime}}=n, . \quad \text {. . . . . . }
$$

where $n$ represents the ratio of the number of bands in the interval $\delta \lambda$ in the two cases.

Hence

$$
\frac{\delta\left(\mathrm{N}-\mathrm{N}^{\prime}\right)}{\frac{\delta \lambda}{\frac{\delta \mathrm{N}}{\partial \lambda}}}=\frac{d-d^{\prime}}{d}=\frac{n-1}{n} \cdot . \cdot . \cdot
$$

Since when $c$ diminishes the bands more in the negative direction, i.e. towards the violet, as shown by equation (10), the total number passing any point, say the sodium line, for a change of path from $d$ to $d^{\prime}$ will be

$$
\begin{aligned}
-m=\int_{d}^{d^{\prime}} \frac{\delta N}{\delta} \delta d & =\int_{d}^{d^{\prime}} \delta d \frac{1}{\lambda}\left(\mathrm{A}+\frac{\mathrm{B}}{\lambda^{2}} \ldots\right) \\
& =\mathrm{N}^{\prime}-\mathrm{N}=\left(d^{\prime}-d\right)\left(\frac{\mathrm{A}}{\lambda}+\frac{\mathrm{B}}{\lambda^{3}}-\frac{\mathrm{C}}{\lambda^{3}}-\delta \lambda\right) .
\end{aligned}
$$

Hence

Hence

$$
\frac{\mathrm{N}-\mathrm{N}^{\prime}}{\mathrm{N}^{-}}=\frac{d-d^{\prime}}{d}=\frac{m}{\mathrm{~N}}=\frac{n-1}{n} . . .
$$

$$
\mathrm{N}=\frac{m n}{n-1}
$$

which gives the order. For example, if $n=2, \mathrm{~N}=2 m$, i. e., if every alternate band of the first coincides with each one in succession of the second, the number of passages necessary to obtain this coincidence from the original complete order of coincidence is one-half the original order of the plate whose thickness is varied. In the substances examined the order for sodium light was approximately double the number of bands easily seen between the red and the blue in the primitive spectrum. For example, in starting with about eight bands visible in the field, fifteen passages for the sodium line were counted in doubling the order, thus making the original order fifteen. Although increase in thickness increased the number of visible bands, the order could be determined to a certainty.

If we call $\mathrm{N}$ the order of the sodium band, and assume it 
to be the central band in the normal spectrum, then, as the sodium band was double the numbar of visible bands, we have approximately $\frac{3 \mathrm{~N}}{4}$ and $\frac{5 \mathrm{~N}}{4}$ as the orders of the red and the violet respectirely. If now we make the order for sodium $n \mathrm{~N} \pm 1$ where $n$ may be a whole or fractional quantity, the corresponding order fo other parts of the spectrum will be increased in the ratio of $\frac{n \mathrm{~N} \pm 1}{\mathrm{~N}}$. Thus for the rell it becomes

$$
\frac{3}{4} \mathrm{~N}\left(\frac{n \mathrm{~N} \pm 1}{\mathrm{~N}}\right)=\frac{3}{2} n \mathrm{~N} \pm \frac{3}{4},
$$

and for the violet

$$
{ }_{4}^{5} \mathrm{~N}\left(\frac{n \mathrm{~N} \pm 1}{\mathrm{~N}}\right)=\frac{5 n \mathrm{~N}}{4} \pm \frac{5}{4} .
$$

This shows that when the order for the sodium band is increased $n$ times by increasing the thickness of the plate $n$ times, a single passage in the positive or negative direction, changing the order to $n \mathrm{~N} \pm 1$, gives a difference of coincidence between the original band of the one spectrum and the nearest band of the other of one-fourth the distance between the new bands, or $\frac{1}{4 n}$. As 01 band can under the most favourable conditions be distinguished, $n=25$ will represent the limit of the ratio of the thicknesses which may be used by this method. The same method and reasoning apply in determining the order and the limit of error for any other part of the spectrum.

The following arrangement (fig. 1) was used for making direct comparisons between two crystalline plates.

Two slits $S$ and $S^{\prime}$ were used for producing two prismatic spectra at $\mathrm{B}$ and $\mathrm{B}^{\prime}$ above each other. The adjustment of the total reflecting prism allowed the spectral lines to be brought into corresponding positions above each other in both spectra. The two crystalline specimens-usually single wedges-were each mounted in an ordinary Babinet's compensator. The wedges were adjusted so that the incident light was normal. The slits were placed immediately after the wedges, and were made as narrow as possible consistently with the bands remaining visible. The cross-hair of the observing telescope was made to coincide with one of the sodium lines in each spectrum. Since the opening of the slits $\mathbf{S}$ and $\mathbf{S}^{\prime}$ and also the angle of the wedges were small, the thickness $d_{1}$ and $d_{2}$ in each wedge may be considered constant. The 
equation of condition ( $2 \alpha$ ) relates only to order and wavelength, hence no attention need be given to the determination

Fig. 1.

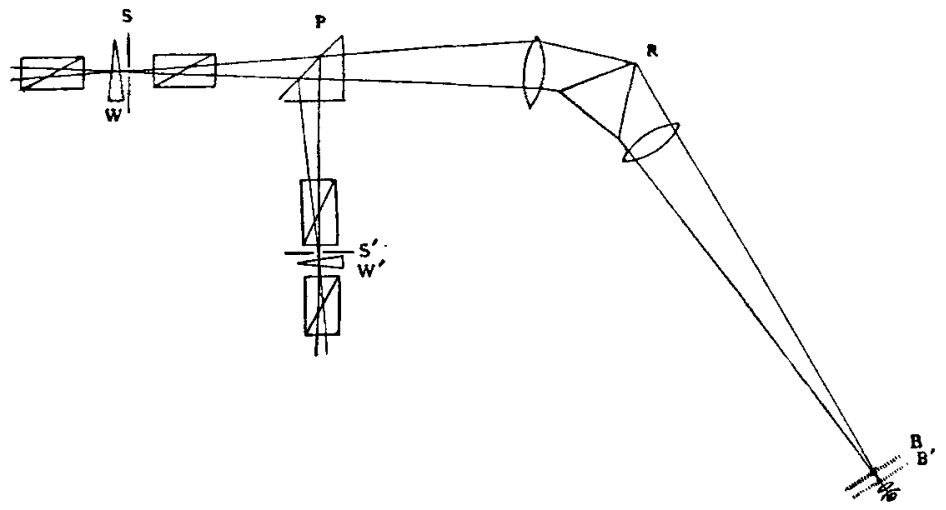

of $d_{1}$ and $d_{2}$ in observing whether achromatism exists. A motion of the micrometer-screw of each compensator before the slit caused the bands to move across the spectrum from the violet to the red end, more coming in at the violet than disappeared at the red end, thus causing the bands to crowd together, while when the screw was moved so as to diminish the thickness, more bands disappeared at the violet end than came into the field at the red end, thus causing a spreading out of the bands, which confirms the conclusions above arrived at. When a prism was used at $R$ the distance between the bands increased from the red toward the violet owing to the greater dispersion of the glass for this part of the spectrum than the differential double refraction of the crystals. It was found that for the values of $d$ used the order of the plate could be determined to a certainty according to the method indicated under equation (15). This obviated the difficulty hitherto met in determining the order of the plate by measuring its thickness and index, which may introduce serious errors, as indicated above in the discrepancies between the results of Mouton and Dufet. It furthermore did not require the use of wedges perfeetly plane, which it is not always possible to obtain, as, for example, in the case of mica and other easily cleavable crystals, accurate data for which are lacking.

Plane parallel plates and wedges of mica, selenite, right and left-handed quartz, Iceland spar, and aragonite were 
made for me by Bernard Halle of Berlin. Through the courtesy of President Kohlrausch I was enabled to make the comparison of the crystalline plates at the Reichsanstalt in Charlottenburg.

\section{Comparison of Right- and Left-handed Quartz.}

Previous direct measurements of $\epsilon$ and $\omega$ in right- and left-handed quartz indicate slight discrepancies, probably instrumental errors. This method of comparison gives a very sensitive test as to the interesting question of differential double refraction in the two kinds of erystals. Accordingly, wedges cut with their lengths parallel to the optic axis were placed before $S$ and $S^{\prime}$, and the latter illuminated with sodium light, so that the path of the ray was normal to the optic axis within the crystal, and adjustment made for coincidence of the cross-hair and the sodium line in the two spectra. This gave perfect coincidence throughout the spectrum, adjustment being made so as to bring the cross-hair on one of the black bands, about ten bands being visible in the field. One of the micrometer-screws was then turned so as to increase the thickness of the wedge, and the number of bands passing the cross-hair toward the red was counted until perfect coincidence of every alternate band in this spectrum with the bands of the former was obtained. The number of black bands counted-namely, twenty-gave the original order for the sodium line. The second wedge was then shifted in the same way until perfect coincidence was obtained, the number o passages across the sodium line being the same as in the first case, namely twenty.

This comparison was extended to the fifty-eighth order for sodium (as high as available with the wedges at hand) and not the slightest deviation from perfect coincidence thronghout the spectrum could be observed. The variation of one passage would destroy the coincidence in a marked degree, so that this method of comparison gives a simple and positive method of determining the order. Different ratios of coincidences, as $2: 1,3: 1,4: 1,3: 2,1: 2,2: 3, \ldots$ were used, and the same orders were always obtained when substituted in equation (15). It may be concluded, therefore, that the differential double refraction in quartz is the same whether it be right- or left-handed.

\section{Comparison of Selenite and Left-handed Quartz.}

In the comparison of selenite and quartz the previous wedge of left-handed quartz was used, and also a wedge of Phil. Mag. S. 5, Vol. 48. No, 293. Oct, 1899, 2 C 
selenite of the thirty-third order cut with its edge parallel to one of the principal axes, one face being in the plane of principal cleavage. Adjustment was made so as to give three bands in the field. This gave almost perfect coincidence of the two sets of bands. On increasing the thickness the approximate coincidence between the alternate bands was obtained by five passages of the sodium line, thus showing the original order to be the fifth. On increasing the thickness of the other wedgo so as to give five passages the approximate coincidence of the bands was again obtained, those of the quartz being shorter than those of the selenite in the blue. By continuing this process of increasing the order of each up to the thirty-second (the limit of the selenite wedge) this shortening of the quartz in the blue increased, the red bands extending beyond those of the selenite. On introducing a plate of selenite before the wedge and adjusting for coincidence, and then increasing the orders successively up to the sixtieth, it was found that a better coincidence in the yellow and toward the blue was obtained on increasing the order of the selenite by one at the forty-ninth order, making the order fifty for selenite and forty-nine for quartz; this, however, increased the disagreement in the red still more. Achromatism was apparently not possible except in parts of the spectrum, the ratio depending on the portion of the spectrum, the reciprocal of this ratio being even possible for the red, say. No anomalous distribution of the bands of selenite could be detected in the region studied by Mouton and Dufet, namely $491 \mu$, where the bands of the selenite could be made to coincide with those of the quartz.

\section{Comparison of Mica and Left-handed Quartz.}

Considerable difficulty was experienced in obtaining a wedge of mica. One of the twenty-eighth order was, however, finally ground by closely cementing together two or three pieces upon a plate of glass and careful polishing. The angle was $6^{\circ} 7^{\prime}$, the edge being parallel to one of the principal axes and one face that of principal cleavage. High orders could not be used on account of the great absorption of light. The quartz wedge was the same as previously used.

Starting with four bands visible in the field, which was as low as comparisons could be made, and increasing the thickness of each to one passage of the sodium line, the best coincidence for the central band was obtained with orders of eight quartz and nine mica, the orders being determined as above by doubling the number of bands in one spectrum. The 
orders seven quartz and eight mica gave also very good coincidence, better than nine quartz and ten mica, which might indicate a ratio between the first two ratios but nearer the first, namely, eight quartz and nine mica. For these orders the quartz bands were slightly closer than those of the mica in the extreme blue, but somewhat more extended in the red. On increasing the thickness of each and noting the passages of the sodium band a second coincidence was obtained for the orders fifteen quartz and seventeen mica, and also for sixteen quartz and eighteen mica. The next approximate coincidence was obtained with twenty-three quartz and twenty-six mica, and also a closer one with twenty-four quartz and twenty-seven mica, the latter giving more complete coincidence for the central band. The orders eight quartz and nine mica were estimated to give the best ratio for achromatism, although each may be slightly too great. The coincidence of the bands was not as perfect, however, as with the selenite and the quartz for the same orders, the deviation of the quartz being at least one-tenth of a band within that of the mica in the blue and extending two-tenths in the red beyond those of the mica.

\section{Comparison of Mica and Selenite.}

The wedges and plates of mica and selenite used previously in the comparisons with quartz were compared directly. With four bands in the field a first coincidence was obtained, the order for each crystal being found to be eight for mica and seven for selenite. The selenite bands in the blue were only perceptibly shorter and also longer in the red. On increasing the orders of each successively a second coincidence was obtained for the sixteenth order of the mica and the fourteenth of the selenite. A closer coincidence was obtained here than for the second coincidence of the mica and quartz. A third coincidence was obtained for the twenty-fourth order of mica and the twenty-first order of selenite. Only slight discrepancies could be observed, the coincidence being almost perfect in the yellow and green. The ratio of eight mica to seven selenite being undoubtedly very approximately the best achromatism.

\section{Comparison of Iceland Spar and Left-ñanded Quartz.}

Comparisons were made with these two crystals, but difficulty was experienced in abtaining a wedge of the former of sufficiently low order and good definition to make accurate $2 \mathrm{C} 2$ 
observations. Two observations, however, were made giving coincidence for the twenty-fourth order of Iceland spar and twenty-fifth order of quartz. A third observation under more favourable conditions gave good coincidence for the orders forty-four Iceland spar and forty-six quartz. The ratio of twenty-two Iceland spar to twenty-three left-handed quartz was selected as the best for achromatism, but further experiments will be necessary to confirm this result.

\section{Comparison of Aragonite and Left-handed Quartz.}

Small wedges were made of aragonite, but the bands were too indistinct to determine their ratios. However, with a wedge whose edge bisected the greater angle between, and whose face was normal to, the plane of the optic axes good coincidence was obtained in the region of the thirtieth to the fifty-eighth order of quartz throughout the spectrum. With a similar wedge, but with its face parallel to this plane, no definite comparisons could be obtained. The determinations for Iceland spar and aragonite have been reserved for future observation.

If we wish to use a combination of two crystals which have not been directly compared their ratio can be easily determined provided coincidences have been obtained over the same portion of the spectrum. Thus the ratio of the orders of mica to selenite is $8: 7$, and of mica to quartz $9: 8$. Hence the ratio of selenite to quartz would be $9: 8:: 8: 7$ or $63: 64$; which would be possible in a part of the spectrum, as stated above.

The results of these observations show that with the more available crystals achromatism cannot be obtained over the entire visible spectrum, but that certain pairs of crystals will achromatize more perfectly than others. For example, better coincidences were obtained with selenite and mica than with quartz and mica. These two pairs are particularly suitable over the others in making compound retardation plates, such as achromatic quarter-wave plates, and the orders used are comparatively low.

Having tound the linear relation between $\mathrm{N}_{1}, \mathrm{~N}_{2}, \ldots$ and satisfied equation ( $2 a$ ) for a part or the whole of the spectrum, as the case may be, we can solve equation $(1 a)$ for any given resultant retardation $\mathrm{N}$ of several crystals. For example, if $N=\lambda$ for mica and selenite we have

$$
\begin{gathered}
N_{M}-N_{8}=\lambda, \quad \frac{N_{M}}{N_{S}^{\prime}}=\frac{8}{7}, \quad N_{M}^{\prime}-\frac{7}{8} N_{M}=\lambda, \\
\therefore \quad N^{2}=8 \lambda \text { and } N_{S}=7 \lambda .
\end{gathered}
$$




$$
\begin{array}{ll}
\mathrm{N}_{\mathrm{M}}-\mathrm{N}_{\mathrm{s}}=\mathrm{N}=\frac{\lambda}{4}, & \frac{\mathrm{N}_{\mathrm{M}}}{\mathrm{N}}=\frac{8}{7}, \\
\therefore \quad \mathrm{N}_{\mathrm{M}}=2 \lambda \text { and } & \mathrm{N}_{\mathrm{S}}=1 \frac{3}{4} \lambda .
\end{array}
$$

If $N=\frac{\lambda}{4}$ for mica and quartz we have

$$
\begin{gathered}
\mathrm{N}_{M}-\mathrm{N}_{Q}=\frac{\lambda}{4}, \quad \frac{\mathrm{N}_{M}}{\mathrm{~N}_{Q}}=\frac{9}{8}, \quad \mathrm{~N}_{M}-\frac{8}{9} \mathrm{~N}_{M}=\frac{\lambda}{4}, \\
\therefore \mathrm{N}_{M}=2 \frac{1}{4} \lambda \quad \text { and } \quad \mathrm{N}_{Q}=2 \lambda .
\end{gathered}
$$

Similarly if $\mathrm{N}=\frac{\lambda}{2}$ for the same pair we have

$$
\mathrm{N}_{\mathrm{M}}=4 \lambda \text { and } \mathrm{N}_{\mathrm{g}}=3 \frac{1}{2} \lambda \text {. }
$$

If $\mathrm{N}=\lambda$ and we cross quartz with mica and selenite we have $\mathrm{N}_{M}-\mathrm{N}_{Q}+\mathrm{N}_{\mathrm{B}}=\lambda$, and therefore

$$
\mathrm{N}_{\mathrm{M}}\left(1-\frac{8}{7}+\frac{7}{8}\right)=\lambda, \text { or } \quad \mathrm{N}_{\mathrm{M}}=\frac{72}{71} \lambda \text {, }
$$

hence

$$
\mathrm{N}_{\mathrm{Q}}=\frac{64}{71} \lambda \text {, and } \mathrm{N}_{\mathrm{s}}=\frac{63}{71} \lambda \text {. }
$$

If we cross selenite with mica and quartz we have in the same way

$$
\mathrm{N}_{\mathrm{M}}=\frac{72}{73} \lambda, \quad \mathrm{N}_{\mathrm{Q}}=\frac{64}{73} \lambda, \quad \text { and } \quad \mathrm{N}_{8}=\frac{63}{73} \lambda .
$$

Thus we see that an eighth order mica and a seventh order selenite crossed give a resultant plate of the first order for all colours approximately, and also that a second order mica and a one and three quarter order selenite will give an achromatic quarter-wave plate. Similarly a ninth order mica and an eighth order right- or left-handed quartz give when crossed an approximately achromatic $1 \lambda$ plate, and a second order quartz and a two and one quarter mica give an approximately achromatic $1 / 4 \lambda$ plate, but less perfectly compensated than with the other pair of crystals. A slight variation from these ratios will give corresponding results providing the difference is accurately $1 \lambda$ or $1 / 4 \lambda$, as the case may be.

\section{Comparison by means of Cross Plates.}

In order to observe the achromatizing effect of crossing two crystals with different ratios of the order, wedges were cut so that when they were superposed with their edges at 


\section{Prof. D. B. Brace on Achromatic Polarization}

right angles they were in subtractive positions. The bands thus formed were seen diagonally across the faces of the wedges, the dark band usually used as a test of the order of a plate thus covering different orders in each wedge. The degree of blackness and of decolorization of this band was an indication of the amount of achromatism.

In order to give a greater range to the observation without cutting a number of wedges of successively higher orders compound wedges̄ were used, 'see fig. 2.

Fig. 2 .

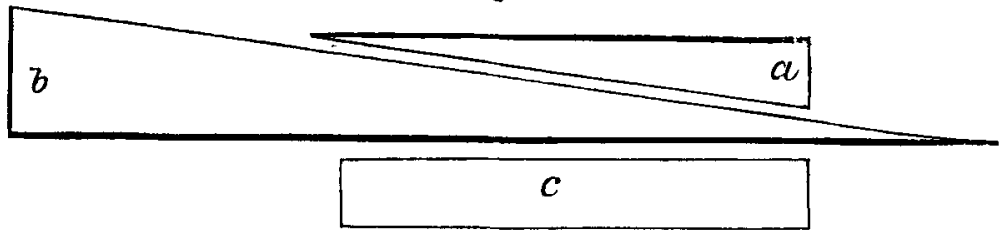

Thus $a$ and $b$ were two wedges cut so that when superposed in the reverse direction they bore the same relation to the principal axes. The angle of $a$ was made slightly different from $b$ so that the system formed a compound wedge of small angle, over which several bands of varying order might be formed by sliding $b$ with respect to $a$. By using $c$ either in subtractive or in additive order, the lowest orders up to the highest could be examined. On placing $a$ and $b$, with or without c, over a corresponding system of another crystal, broad bands could be obtained by proper adjustment of orders up to several hundred or a thousand. On placing selenite over quartz a perfectly colourless dark band was obtained for the zero and lowest orders, but this became gradually more and more coloured as the orders increased up to the fiftieth, a decidedly bluish band being obtained at this point, this indicating compensation for the red end of the spectrum, which is in agreement with the observations made by the first method, while the adjacent higher band was bordered by a pink and green and showed scarcely any blue, indicating compensation for the blue as found previously. With still higher multiples the coloration was increased.

Iceland spar and quartz showed a nearly black line with a bluish tint for the orders twenty-two Iceland spar and twentythree quartz respectively, indicating fairly good achromatism. Multiples of these gave greater coloration, but less than the selenite and quartz for similar orders. 
Iceland spar with selenite gave nearly as dark a band as with quartz at approximately the same orders.

Mica and quartz gave the blackest line for the orders nine and eight respectively, very little coloration being present for these orders.

Mica and selenite also gave the best compensation for the orders eight and seven respectively, less coloration being observable than with the previous pair. These results all agree with those obtained by the previous method.

It should be noted that when the comparisons are made between wedges beginning with the zero order, say, and increasing, that the black band changes into a bright colourless band after a gain of half an order of one of the wedges over the other, and then changes into a black band after a gain of one order, and so on. By counting the number of bands gained it is evident that we can determine the ratios for achromatism for the entire spectrum or for a part, by counting these passages of either the dark or bright bands, the wedges having been previously graduated for the bands of sodium light, say, and the position of either of these bands with respect to the latter determining the ratio. For example, with wedges of selenite and of mica the bright bands occurred at the fourth order in the mica, and the three and one half order in the selenite wedge; and at the eighth and seventh orders respectively, for the first black band gained. Similar results were noted for the other specimens examined.

It is evident from the above that in determining the order of a plane plate by means of a wedge, as is usually done, that the order will depend upon the comparison wedge used. For example, using white light, a mica plate tested with a mica wedge gave $9 \cdot 2$ as the order. The same plate tested with a quartz wedge gave 8.3 as the order, and with a selenite wedge 8.4. A selenite plate gave $24 \cdot 6$ as the order when tested with a selenite wedge, and 28 with a mica wedge. The same results would also follow with measurements made with the ordinary Babinet's compensator.

Of the crystals examined above combinations of mica and selenite, and of mica and quartz, are the most available for retardation plates, such as achromatic quarter-wave plates. Both of these combinations were tried. In the construction of a mica-selenite $1 / 4 \lambda$ plate, for example, a film of mica was split off of as near the second order for sodium as possible. A piece of selenite was then cemented over this in subtractive position and then polished and tested until the resultant retardation was reduced to exactly $\lambda / 4$ for sodium light. On 
placing this pair with their principal axes at $45^{\circ}$ to the polarizer and rotating the analyser, the field appeared white and of uniform intensity, the variation being very much less than with a true $1 / 4 \lambda$ plate of mica or of selenite, but not so perfect as was obtained with the Fresnel rhomb. Fresnel's formula gives for crown-glass a difference of phase of $1 / 10 \lambda$ between the extreme red and violet rays when the relative retardation is one $\lambda$.

When these combinations were examined in divergent light with the polarizer and analyser either crossed or parallel, the isochromatic lines in the centre of the field were nearly colourless, and the neutral hyperbolic line became pink when the line of the optic axes was not parallel or perpendicular to the principal plane of the polarizer ; both sets of fringes, however, had the same general form as when mica was used alone.

Similar results were obtained with mica and quartz, this latter combination not giving quite as good compensation as the former.

Achromatic wedges might also be obtained by a similar combination, the proper ratio of the orders being maintained over the entire length of the wave. Combinations of such systems might also be used as an achromatic compensator in a similar way to that of Babinet's compensator, the distance apart of the bands within the field being given in the equations already discussed.

With systems of more than two crystals better achromatism would be possible, but the mechanical difficulties would be serious. Over parts of the spectrum very close achromatism may be obtained with the above crystals.

Further observations on other crystals may reveal better combinations than already examined. A careful study of the distribution of the bands of different crystals in a normal spectrum would furnish more definite data than the observations here given with a prismatic spectrum.

Physical Laboratory, University of Nebraska, Lincoln, July 22, 1899.

XXXIX. Thorium Radiation. By R. B. Owess, E.E., Tyndall Fellow, Columbic University, New York; Macdonald Professor of Electrical Engineering, McGill University, Montreal*.

THE nature of the radiations emitted by uranium and its 1 compounds was studied in 1896-97 by its discoverer

* Communicated by Prof. J. J. Thomson, F.R.S. 